\title{
On $p$-Adic Zeta-Functions Associated to the Positive Topology of Algebraic Number Fields
}

\author{
By
}

Alexander ScHMIDT*

In [S] we introduced a new Grothendieck topology (called positive topology) over the rings of integers of algebraic number fields. If $K$ is a finite extension of $\boldsymbol{Q}$ then $\operatorname{Spec}\left(\mathcal{O}_{K}\right)$ furnished with the positive topology shows a behavior very similar to the étale site over a complete curve over a finite field.

In this paper we are going to investigate $p$-adic zeta functions associated to the positive topology. Following the analogy it is natural to expect a functional equation. The aim of this paper is to show that such a functional equation holds true as soon as a primitive $p^{\text {th }}$ root of unity is contained in $K$.

If $K / k$ is an abelian extension then the positive $p$-adic zeta-function of $K$ splits into a product of positive $p$-adic L-functions attached to the characters of $K / k$ in the usual way. If the field $k$ is totally real and "totally $p$-real" (e.g. $k=Q$, see below for the definition) we calculate (at least up to the Greenberg conjecture) the positive $p$-adic L-functions $L_{p}^{p o s}(s, \psi)$ in terms of the analytic $p$-adic L-functions defined by Kubota/Leopoldt and Deligne/Ribet.

\section{$\S 1$. Review of the Positive Topology}

In this section we want to summarize the basic definitions and results about the positive topology over the rings of integers of algebraic number fields. Details and proofs can be found in [S]. Let $p$ be a prime number. We

Communicated by Y. Ihara, March 4, 1996.

1991 Mathematics Subject Classification(s): 11R23, 11R42

* Universität Heidelberg, Mathematisches Institut, Im Neuenheimer Feld 288, D-69120 Heidelberg, Germany 
denote the maximal Galois extension of odd degree (resp. p-power degree) of a field $k$ by $k$ (odd) (resp. $k(p)$ ). If $k$ is a local field the maximal unramified extension of $k$ is denoted by $k^{n r}$.

Definition. A field $k / \mathbb{Q}_{p}, p$ odd, is preorientable if $k \subset \mathbb{Q}_{p}^{p r e}:=\mathbb{Q}_{p}^{n r}\left(\zeta_{p}\right.$ $\left.+\zeta_{p}^{-1}\right)($ odd $)\left(\zeta_{p}\right)$.

Example. Every abelian extension $\mathbb{Q}_{p}(p$ odd) is preorientable.

The subgroup $\langle\rho\rangle=\operatorname{Gal}\left(Q_{p}^{\text {pre }} / \mathcal{Q}_{p}^{n r}\left(\zeta_{p}+\zeta_{p}^{-1}\right)(\right.$ odd $\left.)\right) \cong \mathbb{Z} / 2 \mathbb{Z}$ is a normal subgroup of $\operatorname{Gal}\left(Q_{p}^{p r e} / Q_{p}\right)$. Therefore the automorphism $\rho$ acts on every preorientable field. The involution $\rho$ extends the automorphism: $Q_{p}\left(\zeta_{p}\right) \rightarrow Q_{p}\left(\zeta_{p}\right) ; \zeta_{p} \mapsto \zeta_{p}^{-1}$ and we think of it as a local analog of the complex conjugation. One observes that $Q_{p}^{p r e}$ is the maximal extension of $Q_{p}$ having such an involution in a natural way.

Definition. Let $k / Q_{p}, p$ odd, be preorientable. The field of $\rho$-invariant elements in $k$ is denoted by $k^{+}$. We say that $k$ is orientable if either $k=k^{+}$or if $\zeta_{p} \in k^{n r}$.

According to technical problems with the prime number 2 (the Hilbert symbol is in general not alternating) we make the convention that no 2-adic number field is (pre)orientable.

Definition. An extension $L / K$ of algebraic number fields is called positively ramified (p.r.) at the prime $p \mid p$ of $L$ if $L_{\mathfrak{p}} \subset Q_{p}^{\text {pre }} K_{\mathfrak{p}}\left(\subset \bar{Q}_{2}\right.$ if $\left.p=2\right)$ and if the Galois closure $\hat{L}_{\mathfrak{p}} / K_{\mathfrak{p}}$ of $L_{\mathfrak{p}}$ over $K_{\mathfrak{p}}$ has at most pure wild ramification.

This leads to a covering type (the positive coverings) over the rings of integers of algebraic number fields and defines a Grothendieck topology which is finer than the étale topology, i.e. étale coverings are p.r..

If $K / Q$ is a finite number field then there are only finitely many primes $\mathfrak{p}$ of $K$, such that the completion $K_{\mathfrak{p}}$ is not orientable. These can be thought as primes where $\operatorname{Spec}(K)$ has "bad reduction", since the local duality pairing associated to $\operatorname{Spec}\left(\mathcal{O}_{K_{\mathfrak{p}}}\right)_{\text {pos }}$ is degenerate at these primes (see [S] Thm. 3.7). By $d(K)$ we denote the smallest positive integer, such that $K_{\mathfrak{p}}$ is orientable at all 
primes $p$ not dividing $d(K)$. We always have $d(K) \mid 2 \operatorname{disc}(K / Q)$ but usually $d(K)$ is much smaller. If $K$ is a cyclotomic field then $d(K)=2$.

The cohomology groups of sheaves of abelian groups over the positive topology are denoted by $H_{p o s}^{*}$. These satisfy the following global duality theorem, which is the exact analog to the étale Poincare duality theorem for complete curves over finite fields.

Theorem 1 ([S] Thm.1). Let $K$ be a number field and $X=\operatorname{Spec}\left(\mathcal{O}_{K}\right)$. For every integer $n$ with $(n, d(K))=1$ there is a canonical trace map tr: $H_{p o s}^{3}\left(X, \mu_{n}\right)$ $\stackrel{\sim}{Z} / n \boldsymbol{Z}$ and for every locally constant constructible sheaf $F$ of $\boldsymbol{Z} / n \mathbb{Z}$-modules on $X_{\text {pos }}$ the cup-product:

$$
H_{\text {pos }}^{i}(X, F) \times H_{\text {pos }}^{3-i}\left(X, \mathscr{H} \text { om }\left(F, \mu_{n}\right)\right) \rightarrow H_{\text {pos }}^{3}\left(X, \mu_{n}\right) \stackrel{\sim}{\rightarrow} Z / n Z
$$

induces a perfect pairing of finite groups for all $i$.

If $K$ is a number field and $p$ is a prime number then the cyclotomic $Z_{p}$-extension $K_{\infty} / K$ is always positively ramified. For the positive topology these extensions play a similar role as the constant field extensions for function fields. The positive $p$-adic zeta functions will be defined by the asymptotic behavior of the positive cohomology groups going up the cyclotomic tower.

For later use we recall the following definition from [S].

Definition. Let $K$ be a number field an let $p$ be a prime number with $(p, d(K))=1$. We say that

(i) $K$ is totally $p$-real if for every prime $p$ dividing $p$ of $K$ it holds: $K_{\mathfrak{p}}$ $=K_{\mathfrak{p}}^{+}$,

(ii) $K$ is totally $p$-imaginary if $\left[K_{\mathfrak{p}}: K_{\mathfrak{p}}^{+}\right]=2$ for every prime $\mathfrak{p}$ of $K$ dividing $p$,

(iii) $K$ is a $p-\mathbf{C M}$ field if it is totally $p$-imaginary and if it contains a totally $p$-real subfield $K^{+, p}$ of index 2. By $r_{p}(K)$ we denote the number of complex places of $K^{+, p}$.

In order to prevent confusion a number field which is real, resp. $\mathrm{CM}$ in the usual sense will be called $\infty$-real, resp. $\infty-\mathrm{CM}$ in the following. A number field $K$ which is $\infty$-CM may or may not be $p$-CM and vice versa. If $K$ is both $\mathrm{CM}$ and $p$-CM the $(+)$-fields may or may not be the same. 
If $K$ is $p$-CM then the action of the " $p$-complex conjugation" decomposes the cohomology of $X=\operatorname{Spec}\left(\mathcal{O}_{K}\right)$ in the following way. Here we denote by $S_{p}$ the set of primes of $K$ dividing $p$ :

$$
H_{p o s}^{1}\left(X, \mathbb{Q}_{p} / \mathbb{Z}_{p}\right)=H_{e t}^{1}\left(X \backslash S_{p}, \mathbb{Q}_{p} / \mathbb{Z}_{p}\right)^{+} \otimes H_{e t}^{1}\left(X, \mathbb{Q}_{p} / \mathbb{Z}_{p}\right)^{-} .
$$

\section{§2. Positive $p$-Adic Zeta Functions}

In this section we fix a number field $K$ and an odd prime number $p$. We denote the cyclotomic $\mathbb{Z}_{p}$-extension of $K$ by $K_{\infty}$.

In order to define a $p$-adic zeta function for the positive topology on $X$ $=\operatorname{Spec}\left(\mathcal{O}_{K}\right)$ we consider the action of a fixed generator $\gamma$ of $\Gamma=\operatorname{Gal}\left(K_{\infty} / K\right)$ on the positive cohomology of the normalization $X_{\infty}$ of $X$ in $K_{\infty}$. We think of $\gamma$ as an arithmetic Frobenius automorphism. We denote the subextension of degree $p^{n}$ in $K_{\infty}$ by $K_{n}$ and the normalization of $X=\operatorname{Spec}\left(\mathcal{O}_{K}\right)$ in $K_{n}$ by $X_{n}$.

One is tempted to define the $p$-adic positive cohomology groups of $X_{\infty}$ as the projective limit of $H_{p o s}^{i}\left(X_{\infty}, \mathbb{Z} / p^{n} \mathbb{Z}\right)$, however these cohomology groups are finite only under very restrictive conditions (e.g. if $K$ is $p$-CM, $r_{p}(K)=0$ and the Iwasawa $\mu$-invariant of $K$ vanishes) and therefore this limit would not define a compact $\Lambda$-module in general.

If $C$ is a complete, smooth curve over a finite field $k(\operatorname{char}(k) \neq p)$ then étale Poincaré duality gives the following alternative description: $H_{e t}^{i}\left(\bar{C}, \mathbb{Z}_{p}(k)\right)$ $=H_{e t}^{2-i}\left(\bar{C}, \mathbb{Q}_{p} / \mathbb{Z}_{p}(1-k)\right)^{*}$. (Here $A^{*}$ denotes the Pontrjagin dual of a locally compact abelian group $A$.) This motivates the following definition for the positive topology:

Definition. We call the groups

$$
H_{p o s}^{i}\left(X_{\infty}, \mathbb{Z}_{p}(k)\right) \stackrel{d f}{=} H_{p o s}^{2-i}\left(X_{\infty}, Q_{p} / Z_{p}(1-k)\right)^{*}
$$

the $p$-adic positive cohomology groups of $X_{\infty}$ with values in $\mathbb{Z}_{p}(k)(k \in \mathbb{Z})$.

Lemma 2.1. The groups $H_{p o s}^{i}\left(X_{\infty}, \mathbb{Z}_{p}(k)\right)$ are finitely generated modules under the Iwasawa algebra $\Lambda=\mathbb{Z}_{p}[[\Gamma]]$ and trivial for $i<0$ and $i>2$.

Proof. Since positive cohomology commutes with projective limits of schemes and inductive limits of sheaves (see [S] Lemma 2.2) we have 


$$
H_{p o s}^{i}\left(X_{\infty}, Q_{p} / Z_{p}(k)\right)=\lim _{\vec{n}, m} H_{p o s}^{i}\left(X_{n}, Z / p^{m} Z(k)\right)
$$

Using the Hochschild-Serre spectral sequence for $X_{\infty} / X$ (see [S] Prop. 2.1) the first statement follows from the finiteness of $H_{p o s}^{i}\left(X, Z / p^{m} Z(k)\right)$. Further these groups vanish for $i<0$ and $i>3$. Therefore it only remains to show the vanishing of $H_{p o s}^{-1}\left(X_{\infty}, Z_{p}(k)\right)$. By the global duality theorem we have

$$
H_{p o s}^{3}\left(X_{n}, Q_{p} / Z_{p}(k)\right)=\lim _{n} H_{p o s}^{0}\left(X_{n}, Z / p^{n} Z(1-k)\right)=0 \text { for } k \neq 1 \text {. }
$$

Therefore

$$
H_{p o s}^{-1}\left(X_{\infty}, Z_{p}(k)\right)=\left(\lim _{\vec{n}} H_{p o s}^{3}\left(X_{n}, Q_{p} / Z_{p}(1-k)\right)^{*}=0 \text { for } k \neq 1\right.
$$

The last statement is also true for $k=1$ as it can be seen from the commutative diagrams for all $n \leq N$ (see [S] Prop. 4.1, Cor. 4.4):

$$
\begin{array}{ccc}
H_{p o s}^{3}\left(X_{n}, \mu_{p^{\infty}}\right) & \stackrel{\sim}{\rightarrow} & Q_{p} / Z_{p} \\
\downarrow^{c a n} & & \downarrow^{\cdot p^{N-n}} \\
H_{p o s}^{3}\left(X_{N}, \mu_{p^{\infty}}\right) & \stackrel{\sim}{\rightarrow} & Q_{p} / Z_{p} .
\end{array}
$$

In the following we will make extensive use of the results of Jannsen's paper [Ja]. For the convenience of the reader we shortly recall the basic notations and results.

Let $M$ be a finitely generated module under the Iwasawa-algebra $\Lambda=Z_{p}[[\Gamma]]$. We will use the following notations (cf. [Ja])

$T_{0}(M) \quad$ the maximal finite $\Lambda$-submodule of $M$, $T_{1}(M) \quad$ the $\Lambda$-torsion submodule of $M$, $M^{\delta} \quad$ the maximal $\Lambda$-submodule of $M$ on which $\Gamma$ acts discretely, i.e. $M^{\delta}=\cup_{n} M^{\Gamma_{n}}$, where $\Gamma_{n}$ denotes the closed subgroup of index $p^{n}$ in $\Gamma$,

$E^{i}(M) \quad \operatorname{Ext}_{\Lambda}^{i}(M, \Lambda)$ for $i=0,1, \cdots$ 
$E^{i}(M)$ is trivial for $i \geq 3$ and finite for $i=2 . \quad E^{0}(M)$ is free of the same rank as $M . E^{1}(M)$ is $\Lambda$-torsion. If $M$ is a $\Lambda$-torsion module then $\mathbb{E}^{1}(M)$ is isomorphic to the Iwasawa-adjoint $\alpha(M)$ of $M$ (see [Ja]).

Let as before $\gamma$ be a fixed generator of $\Gamma=\operatorname{Gal}\left(K_{\infty} / K\right)$. For a $\Lambda$-torsion module $M$ we define the characteristic polynomial by

$$
f(t, M)=f(t, \gamma, M)=p^{\mu(M)} \operatorname{det}\left(1-\gamma^{-1} t ; M \otimes_{\mathbb{Z}_{p}} \mathbb{Q}_{p}\right),
$$

where $\mu(M)$ is the Iwasawa $\mu$-invariant of $M$.

This definition is standard at least up to an invertible constant. However the $p$-adic positive cohomology groups have $\Lambda$-rank $>0$ in general and thus the question for characteristic polynomials for non-torsion $\Lambda$-modules arises. Several authors defined the characteristic polynomial of a non-torsion module to be identically zero in order to hold true the multiplicativity of characteristic polynomials in exact sequences. In this paper we make the following convention:

The characteristic polynomial of a $\Lambda$-module $M$ is defined by

$$
f(t, \gamma, M):=f\left(t, \gamma, T_{1}(M)\right)
$$

By the isomorphism $E^{1}\left(E^{1}(M)\right) \cong T_{1}(M) / T_{0}(M)$ (see [Ja] Lemma 3.7.b) the definition of the characteristic polynomial given above only depends on the homotopy class of the $\Lambda$-module $M$ (cf. [Ja]). Further this is the only possible definition which extends the definition for torsion modules and which has the property that quasiisomorphic as well as homotopic modules have the same characteristic polynomial.

Defimition. We call the function

$$
Z_{p}(X, t)=\prod_{i=0}^{2} f\left(t, \gamma, H_{p o s}^{i}\left(X_{\infty}, Z_{p}\right)\right)^{(-1)^{i+1}}
$$

the positive $\mathbb{p}^{\text {-adic }}$ zeta function of $X$ with respect to $\gamma$.

\section{§3. The Functional Equation}

Fixing the notations of the last section we now assume $(p, d(K))=1$ and 
$\zeta_{p} \in K$. By $q \in Z_{p}{ }^{\times}$we denote the image of $\gamma$ under the cyclotomic character $\kappa: \Gamma \rightarrow \mathbb{Z}_{p}{ }^{\times}$which is defined by the action of $\Gamma$ on the roots of unity of $p$-power order. (In the function field case $q$ is the number of elements in the constant field when $\gamma$ is the arithmetic Frobenius automorphism.) The unit $q$ is a principal unit and we denote the uniquely defined principal unit $\alpha$ with $\alpha^{2}=q$ by $q^{1 / 2}$.

Theorem 2. If $(p, d(K))=1$ and $\zeta_{p} \in K$ then

$$
Z_{p}(X, t)=\frac{f\left(t, H_{p o s}^{1}\left(X_{\infty}, \mathbb{Z}_{p}\right)\right)}{(1-t)(1-q t)}
$$

and $Z_{p}(X, t)$ satisfies the functional equation

$$
Z_{p}\left(X, \frac{1}{q t}\right)=\varepsilon\left(t q^{1 / 2}\right)^{2-\lambda} Z_{p}(X, t),
$$

where $\lambda$ is the Iwasawa- $\lambda$-invariant of $H_{\text {pos }}^{1}\left(X_{\infty}, \mathbb{Z}_{p}\right)$ and $\varepsilon=(-1)^{r}$, where $r$ is the multiplicity of the eigenvalue $q^{1 / 2}$ of $\gamma^{-1}$ acting on the $\lambda$-dimensional $\mathbb{Q}_{p}$-vector space $T_{1}\left(H_{p o s}^{1}\left(X_{\infty}, Z_{p}\right)\right) \otimes_{\mathbb{Z}_{p}} \mathbb{Q}_{p}$.

Remark. Following the analogy to the case of curves over finite fields one is tempted to speculate about a $p$-adic Riemann hypothesis, however so far we do not know, what the precise statement should be. For a first naive approach it would be natural to ask whether for all eigenvalues $\alpha$ of $\gamma^{-1}$ acting on $T_{1}\left(H_{p o s}^{1}\left(X_{\infty}, \mathbb{Z}_{p}\right)\right) \otimes_{\mathbb{Z}_{p}} Q_{p}$ it holds:

$$
\left\|\log _{p} \alpha\right\|_{p}=\left\|\log _{p} q^{1 / 2}\right\|_{p}
$$

If for example $K$ is an abelian number field with $\zeta_{p} \in K$ and $r_{p}(K)=0$ then we will see in the next section (theorem 3 ) that the eigenvalues in question are exactly

$$
q^{s_{1}}, q^{1-s_{1}} \cdots, q^{s_{k}}, q^{1-s_{k}}
$$

where $s_{1}, \cdots, s_{k}$ are the zeros of the (Kubota/Leopoldt) $p$-adic L-functions attached to the even characters of $K / Q$.

Thus equation $(*)$ is equivalent to the fact, that non of the $s_{i}$ is congruent to 0 or 1 modulo $p$, i.e. there are no zeros of the $p$-adic $\mathbb{L}$-function on "the 
boundary of the critical strip". (Recall that $s_{i} \neq 0$ is the finiteness of the class number while $s_{i} \neq 1$ is Leopoldts conjecture.)

By the calculations of Wagstaff (see [Wag1], [Wag2]) this statement is true for $K=Q\left(\zeta_{p}\right)$ with $p<125,000$.

However Washington [Wash] gives examples of biquadratic fields for which the above formulation of a R.H. is false for $p=3$. To mention the smallest counterexample:

the field $K=Q(\sqrt{122}, \sqrt{-3})$ has $h^{+}=2, h^{-}=12$, there is exactly one zero $s_{1}$ of the 3-adic L-function attached to the even quadratic character of $K$ and $s_{1} \equiv 1 \bmod 27$.

The statements of theorem 2 will be easy consequences of the following proposition.

Proposition 3.1. If $\zeta_{p} \in K$ then the following holds for all $k \in \mathbb{Z}$ :

(i) There is a canonical exact sequence

$$
0 \rightarrow \mathbb{Z}_{p}(k) \rightarrow H_{p o s}^{0}\left(X_{\infty}, Z_{p}(k)\right) \rightarrow E^{0}\left(H_{p o s}^{1}\left(X_{\infty}, Z_{p}(1-k)\right)\right) \rightarrow 0 .
$$

In particular: $T_{1}\left(H_{p o s}^{0}\left(X_{\infty}, \mathbb{Z}_{p}(k)\right)\right) \cong \mathbb{Z}_{p}(k)$.

(ii) For $M=H_{p o s}^{1}\left(X_{\infty}, Z_{p}\right)$ there is a canonical exact sequence

$$
0 \rightarrow T_{0}(M(k)) \rightarrow T_{1}(M(k)) \rightarrow E^{1}\left(T_{1}(M(1-k))\right) \rightarrow 0 .
$$

(iii) $H_{p o s}^{2}\left(X_{\infty}, Z_{p}(k)\right) \cong Z_{p}(k-1)$.

Proof. By $E^{j}(N(-k))=E^{j}(N)(k)$ and $T_{j}(N(k))=T_{j}(N)(k)$ for all $j$ and every $\Lambda$-module $N$ all statements of the proposition are invariant under twists and therefore it suffices to show them for one suitable $k$. In the following we choose $k$ such that

$$
\begin{array}{ll}
\text { A } & H^{i}\left(\Gamma, Q_{p} / Z_{p}(k)\right)=0=H^{i}\left(\Gamma, Q_{p} / Z_{p}(1-k)\right) \text { for } i \geq 1, \\
\text { B } & T_{0}\left(H_{p o s}^{1}\left(X_{\infty}, Z_{p}(k)\right)\right)=H_{p o s}^{1}\left(X_{\infty}, Z_{p}(k)\right)^{\delta} .
\end{array}
$$

This is certainly possible since $\mathbf{A}$ is true for all $k \neq 0,1$ and in $\mathbf{B}$ we have to exclude the finitely many $k$ such that $q^{-k} \zeta$, with some $p$-power root of unity $\zeta$, is an eigenvalue of $\gamma$ acting on $T_{1}\left(H_{p o s}^{1}\left(X_{\infty}, Z_{p}\right)\right) \otimes_{\mathbb{Z}_{p}} Q_{p}$. 
By the global duality theorem we have

$$
H_{p o s}^{i}\left(X_{\infty}, Z_{p}(k)\right)=\left(\lim _{n, m} H_{p o s}^{2-i}\left(X_{n}, Z / p^{m} Z(1-k)\right)\right)^{*}=\lim _{n, m} H_{p o s}^{i+1}\left(X_{n}, Z / p^{m} Z(k)\right) .
$$

Now we fix $k$ satisfying conditions $\mathbf{A}$ and $\mathbf{B}$ above. The Hochschild-Serre spectral sequence together with condition $\mathbf{A}$ yields

$$
H_{p o s}^{1}\left(X_{n}, Q_{p} / Z_{p}(k)\right)=H_{p o s}^{1}\left(X_{\infty}, Q_{p} / Z_{p}(k)\right)^{\Gamma} .
$$

The short exact sequences $0 \rightarrow Z / p^{m} Z \rightarrow Q_{p} / Z_{p}(k) \stackrel{\cdot p^{m}}{\rightarrow} Q_{p} / Z_{p}(k) \rightarrow 0$ imply the exact sequences

$$
0 \rightarrow H_{p o s}^{0}\left(X_{n}, Q_{p} / Z_{p}(k)\right) / p^{m} \rightarrow H_{p o s}^{1}\left(X_{n}, Z / p^{m} Z(k)\right) \rightarrow{ }_{p^{m}} H_{p o s}^{1}\left(X_{n}, Q_{p} / Z_{p}(k)\right) \rightarrow 0
$$

and the following exact sequence in the projective limit

$$
0 \rightarrow Z_{p}(k) \rightarrow H_{p o s}^{0}\left(X_{\infty}, Z_{p}(k)\right) \rightarrow \lim _{n, m} p^{m} H_{p o s}^{1}\left(X_{\infty}, Z_{p}(1-k)\right)^{* \Gamma_{n}} \rightarrow 0
$$

By [Ja] Lemma 3.9. we can identify this sequence with the sequence in (i). For the rest of the proof we set: $\left.M=H_{p o s}^{1}\left(X_{\infty}, Z_{p}\right), Y=\lim _{n, m} p^{m} H_{p o s}^{2}\left(X_{n}, Q_{p} / Z_{p}\right)\right)$.

From [Ja] Lemma 3.9. e) and conditions $\mathbf{A}$ and $\mathbf{B}$ it follows:

$$
\begin{aligned}
\lim _{n, m} H_{p o s}^{1}\left(X_{n}, Q_{p} / Z_{p}(1-k)\right) / p^{m} & =\lim _{\tilde{n}, m} H_{p o s}^{1}\left(X_{\infty}, Q_{p} / Z_{p}(1-k)\right)^{\Gamma_{n}} / p^{m} \\
& =\lim _{n, m} M(k)^{* \Gamma_{n}} / p^{m} \\
& =E^{1}\left(M(k) / M(k)^{\delta}\right) \\
& =E^{1}\left(M(k) / T_{0}(M(k))\right) .
\end{aligned}
$$

Therefore the exact sequences

$$
\begin{aligned}
0 \rightarrow H_{p o s}^{1}\left(X_{n}, Q_{p} / Z_{p}(1-k)\right) / p^{m} & \rightarrow H_{p o s}^{2}\left(X_{n}, Z / p^{m} Z(1-k)\right) \\
& \rightarrow{ }_{p^{m}} H_{p o s}^{2}\left(X_{n}, Q_{p} / Z_{p}(1-k)\right) \rightarrow 0
\end{aligned}
$$


imply the exact sequence

$$
0 \rightarrow \mathbb{E}^{1}\left(M(k) / T_{0}(M(k))\right) \rightarrow M(1-k) \rightarrow Y(1-k) \rightarrow 0
$$

in the projective limit. By the Hochschild-Serre spectral sequence we have exact sequences

$$
\begin{aligned}
0 \rightarrow H_{p o s}^{1}\left(X_{\infty}, \mathbb{Q}_{p} / \mathbb{Z}_{p}(1-k)\right)_{\Gamma_{n}} & \rightarrow H_{p o s}^{2}\left(X_{n}, \mathbb{Q}_{p} / \mathbb{Z}_{p}(1-k)\right) \\
& \rightarrow H_{p o s}^{2}\left(X_{\infty}, \mathbb{Q}_{p} / \mathbb{Z}_{p}(1-k)\right)^{\Gamma_{n}} \rightarrow 0
\end{aligned}
$$

By $[\mathrm{Ja}]$ Lemma 3.9. d) and condition $\mathbb{B}$ we have

$$
\lim _{\tilde{n}, m} p^{m}\left(H_{p o s}^{1}\left(X_{\infty}, \mathbb{Q}_{p} / \mathbb{Z}_{p}(1-k)_{\Gamma_{n}}\right)=\mathbb{E}^{1}\left(M(k)^{\delta}\right)=\mathbb{E}^{1}\left(T_{0}(M(k))\right)=0\right.
$$

Further by [Ja] Lemma 3.9. f)

$$
\lim _{n, m}\left(H_{p o s}^{1}\left(X_{\infty}, Q_{p} / \mathbb{Z}_{p}(1-k)_{\Gamma_{n}}\right) / p^{m}=E^{2}(M(k))\right.
$$

Applying the snake lemma w.r.t. $p^{m}$-multiplication to the last exact sequence we obtain the exact sequence

$$
0 \rightarrow Y(1-k) \rightarrow \mathbb{E}^{0}\left(H_{p o s}^{0}\left(X_{\infty}, \mathbb{Z}_{p}(k)\right) \rightarrow \mathbb{E}^{2}(M(k))\right.
$$

Hence $\mathbb{T}_{1}(Y(1-k))=0$.

Therefore we get an isomorphism

$$
E^{1}\left(M(k) / T_{0}(M(k))\right) \cong T_{1}(M(1-k)) .
$$

Applying $E^{1}$ once more the identity (see [Ja] Lemma 3.7.b)i))

$$
E^{1} E^{1}\left(M(k) / T_{0}(M(k))\right) \cong T_{1}(M(k)) / T_{0}(M(k))
$$

completes the proof of (ii).

Eventually (iii) follows from

$$
H_{p o s}^{2}\left(X_{\infty}, \mathbb{Z}_{p}(k)\right)=H_{p o s}^{0}\left(X_{\infty}, Q_{p} / \mathbb{Z}_{p}(1-k)\right)^{*}=\mathbb{Z}_{p}(k-1) .
$$

Remark. Denoting the $\lambda$-invariant of the Galois group of the maximal $p$-abelian unramified outside $p$ (resp. everywhere unramified) extension of $\mathbb{K}_{\infty}$ 
by $\lambda_{1}$ (resp. $\lambda_{2}$ ) one easily verifies the following relations with the $\lambda$-invariant $\lambda$ of $H_{p o s}^{1}\left(X_{\infty}, Z_{p}\right)$ :

(i) If $K$ is totally $p$-real then $\lambda=\lambda_{1}$

(ii) If $K$ is $p$-CM then $\lambda=\lambda_{1}^{+}+\lambda_{2}^{-}$, where $\lambda_{1}^{+}$resp. $\lambda_{2}^{-}$are the $\lambda$-invariants of the corresponding $(+)$ or $(-)$-parts with respect to the " $p$-complex conjugation".

(iii) $\lambda \geq \lambda_{2}$ if $H_{\text {pos }}^{1}\left(X_{\infty}, Z_{p}\right)$ is a $\Lambda$-torsion module.

Proof of theorem 2. From (i) and (iii) of proposition 3.1 it follows:

$$
f\left(t, \gamma, H_{p o s}^{0}\left(X_{\infty}, Z_{p}\right)\right)=(1-t) \text { and } f\left(t, \gamma, H_{p o s}^{2}\left(X_{\infty}, Z_{p}\right)\right)=(1-q t) .
$$

Further by proposition 3.1 (ii) we have for $M:=T_{1}\left(H_{p o s}^{1}\left(X_{\infty}, Z_{p}\right)\right)$

$$
M \sim E^{1}(M(1)) .
$$

Therefore theorem 2 is a formal consequence of the fact that $E^{1}(M(1))$ is isomorphic to the Iwasawa-adjoint of $M(1)$ (see [Ja] Lemma 3.1).

Remark. The eigenvalues of $\gamma^{-1}$ acting on $T_{1}\left(H_{p o s}^{1}\left(X_{\infty}, Z_{p}\right)\right) \otimes_{z_{p}} Q_{p}$ are principal units, in particular the eigenvalue $-q^{1 / 2}$ does not occur. Therefore the above arguments show that the sign $\varepsilon$ of the functional equation is also equal to $(-1)^{\lambda}$. One can show that $\varepsilon$ equals +1 if $K$ is $p-C M$ (e.g. $K / Q$ abelian), or if $H_{p o s}^{1}\left(\operatorname{Spec}\left(\mathcal{O}_{K_{\infty}}\right), Z_{p}\right)$ is a $\Lambda$-torsion module. In fact we do not know any example, when the sign $\varepsilon$ of the functional equation is -1 .

\section{§4. Comparison with Analytic $p$-Adic L-Functions}

Let $p$ be an odd prime number and let $k$ be a number field which is totally $\infty$-real and totally $p$-real. Let $\psi$ be a (one-dimensional) $\bar{Q}_{p}$-valued character for $k$ and let $k^{\psi}$ be the extension of $k$ attached to $\psi$, i.e. $\psi$ is a faithful character $G\left(k^{\psi} / k\right) \rightarrow \bar{Q}_{p}^{\times}$.

In the following we will assume that

(i) $\psi$ is of type $S$, i.e. $k^{\psi} \cap k_{\infty}=k$,

(ii) $H:=k^{\psi}\left(\zeta_{p}\right)$ is $\infty-\mathrm{CM}$ and $p-\mathrm{CM}$.

In the case $k=Q$ condition (ii) is obviously empty. We introduce the following notations: 
$F_{\infty} \quad$ the cyclotomic $Z_{p}$-extension of a number field $F$,

$M_{1}(F) \quad$ the Galois group of the maximal $p$-abelian extension of $F_{\infty}$, which is unramified outside $p$,

$M_{2}(F)$ the Galois group of the maximal $p$-abelian unramified extension of $F_{\infty}$

$X \quad=\operatorname{Spec}\left(\mathcal{O}_{H}\right)$,

$X_{\infty}$ the normalization of $X$ in $H_{\infty}$,

$\Delta \quad=\operatorname{Gal}(H / k)=\operatorname{Gal}\left(H_{\infty} / k_{\infty}\right)$,

$\Delta^{*} \quad$ the character group of $\Delta$,

$\Gamma \quad \operatorname{Gal}\left(H_{\infty} / H\right) \cong Z_{p}$,

$\gamma \quad$ a fixed topological generator of $\Gamma$,

$\omega \in \Delta^{*} \quad$ the Teichmüller character $g\left(\zeta_{p}\right)=\zeta_{p}^{\omega(g)}$ for $g \in \Delta$,

$q \in Z_{p}{ }^{\times} \quad$ the unique principal unit in $Z_{p}{ }^{\times}$, in which is defined by $\gamma\left(\zeta_{p^{n}}\right)=\zeta_{p^{n}}^{q}$

We write $\mathcal{O}_{\psi}$ for $Z_{p}[\psi]$ and for a $Z_{p}[[\Gamma]][\Delta]$-module $M$ we define the $\psi$-part $M^{\psi}$ of $M$ by

$$
M^{\psi}:=\left\{x \in M \otimes_{\mathbf{z}_{p}} \mathcal{O}_{\psi} \mid \sigma(x)=\psi(\sigma) x \text { for all } \sigma \in \Delta\right\} .
$$

This is a $\mathfrak{O}_{\psi}[[\Gamma]]$-module and we define its characteristic polynomial as in the last section by

$$
f\left(t, \gamma, M^{\psi}\right)=\pi^{\mu(M)} \operatorname{det}\left(1-\gamma^{-1} t ; T_{1}(M) \otimes_{\mathbf{z}_{p}} Q_{p}\right),
$$

where $\mu(M)$ is the $\mu$-invariant and $\pi$ is a fixed uniformizer of $\mathcal{O}_{\psi}$.

Definition. We define the positive $p$-adic L-function attached to the character $\psi$ by

$$
L_{p}^{p o s}(s, \psi)=\prod_{i=0}^{2} f\left(q^{-s}, \gamma, H_{p o s}^{i}\left(X_{\infty}, Z_{p}\right)^{\psi}\right)^{(-1)^{i+1}}
$$

This defines a $p$-adic meromorphic function on $Z_{p}$.

By condition (ii) the field $H$ is $p-\mathrm{CM}$ and $\infty-\mathrm{CM}$ and we denote the associated involutions in $\Delta$ by $\rho_{p}$ and $\rho_{\infty}\left(\rho_{\infty}\right.$ is the complex conjugation, while $\rho_{p}$ is the " $p$-complex conjugation".)

Definition. A character $\phi \in \Delta^{*}$ is called $\infty$-odd ( $\infty$-even) resp. $p$-odd (p-even) if $\phi\left(p_{\infty}\right)=-1(+1)$ resp. $\phi\left(\rho_{p}\right)=-1(+1)$. 
An $\infty$-even ( $\infty$-odd) character is even (odd) in the usual sense. It is well known that $M_{1}^{\phi}$ is a $\Lambda$-torsion module if the character $\phi$ is $\infty$-even.

If $\psi$ is $\infty$-even we denote by

$$
L_{p}(s, \psi)
$$

the $p$-adic $L$-function defined by Deligne and Ribet (see [DR]) associated to $\psi$. This function in continuous for $s \in Z_{p}-\{1\}$ and even at $s=1$ if $\psi$ is not trivial. It has the interpolation property

$$
L_{p}(1-n, \psi)=L\left(1-n, \psi \omega^{-n}\right) \prod_{\mathfrak{p} \in S_{p}(k)}\left(1-\psi \omega^{-n}(\mathfrak{p}) N p^{n-1}\right)
$$

for $n \geq 1$, where $S_{p}(k)$ is the set of primes dividing $p$ in $k$ and $L\left(1-n, \psi \omega^{-n}\right)$ is the value of the classical Artin $L$-function $L\left(s, \psi \omega^{-n}\right)$.

If $\psi$ is not $\infty$-even the above interpolation property defines the constant zero function.

For an $\infty$-even character $\psi$ Deligne and Ribet have shown that there exists a unique power series $G_{\psi} \in \mathcal{O}_{\psi}[[T]]$ such that

$$
L_{p}(1-s, \psi)= \begin{cases}G_{\psi}\left(q^{s}-1\right) & \text { if } \psi \neq 1 \\ \left(q^{s}-1\right)^{-1} G_{\psi}\left(q^{s}-1\right) & \text { if } \psi=1\end{cases}
$$

By the main conjecture of Iwasawa theory (proved by Wiles [W] in its most general form over arbitrary totally real fields) there exists an invertible power series $v_{\psi}(T) \in \mathcal{O}_{\psi}[[T]]^{\times}$with

$$
G_{\psi}(T-1)=f\left(T, \gamma, M_{1}(H)^{\psi}\right) v_{\psi}(T-1) \pi^{-\delta_{\psi}},
$$

and the (hypothetical) defect of $\mu$-invariants $\delta_{\psi}$ vanishes if $k=Q$ (be the theorem of Ferrero-Washington) or if the order of $\psi$ is prime to $p$ (see [W] Thm.1.4).

One can reformulate the statement of the main conjecture in terms of the characteristic polynomial of the Iwasawa-module $M_{2}$. The vanishing of the interpolating $p$-adic function for $\infty$-odd characters then motivates the following

Conjecture (Greenberg [G]). $\quad M_{2}(F) \sim 0$ for every totally real number field $F$. 
This conjecture is widely accepted to be true, however, as far as we know, it is verified only in special cases, e.g. if there is only one prime dividing $p$ in $F_{\infty}$ and $\left(h_{F}, p\right)=1$ in which case $M_{2}(F)=0$. For $p=3$ and small degrees $F / Q$ several mathematicians (e.g. $[\mathrm{KS}],[\mathrm{T}]$ ) carried out extensive computations verifying the Greenberg conjecture for many fields.

Theorem 3. There exists an invertible power series $u_{\psi} \in \mathcal{O}_{\psi}[[T]]^{\times}$such that

$$
L_{p}^{p o s}(s, \psi)=\pi^{\delta_{\psi}} u_{\psi}\left(q^{1-s}-1\right) L_{p}(s, \omega \psi) .
$$

if $\psi$ is $\infty$-odd and $p$-odd and

$$
L_{p}^{p o s}(s, \psi)=\pi^{\delta_{\psi}} u_{\psi}\left(q^{s}-1\right) q^{\left(1-\lambda_{\psi}\right) s} L_{p}\left(1-s, \psi^{-1}\right)
$$

if $\psi$ is $\infty$-even and p-even,

where $\lambda_{\psi}$ is the Iwasawa- $\lambda$-invariant of the $\mathcal{O}_{\psi}[[\Gamma]]$-module $H_{p o s}^{1}\left(X_{\infty}, Z_{p}\right)^{\psi}$. Further $\delta_{\psi}=0$ if $k=Q$ or if the order of $\psi$ is prime to $p$.

(iii) Further if the Greenberg conjecture is true then

$$
L_{p}^{p o s}(s, \psi) \equiv 0
$$

if $\psi$ is $\infty$-odd and p-even or $\infty$-even and p-odd.

Remark. Obviously case (iii) only occurs if $\rho_{p} \neq \rho_{\infty}$ or equivalently if $r_{p}(H)>0$.

Corollary 4.1. Assume that the number field $K$ is $p-C M$ with $r_{p}(K)=0$ and $\zeta_{p} \in K$. Then the following holds for the positive p-adic zeta function of $X=\operatorname{Spec}\left(\mathcal{O}_{\mathbf{K}}\right)$ :

$$
Z_{p}\left(X, q^{-s}\right)=q^{\frac{1}{2} s(2-\lambda)} u_{1}\left(q^{s}-1\right) u_{2}\left(q^{1-s}-1\right) \zeta_{p}(s) \zeta_{p}(1-s)
$$

where $\lambda$ is the Iwasawa $\lambda$-invariant of $H_{p o s}^{1}\left(\operatorname{Spec}\left(\mathcal{O}_{K_{\infty}}\right), Z_{p}\right), \zeta_{p}(s)$ is the $p$-adic zeta-function of the maximal totally real subfield $K^{+}$of $K$ and $u_{1}, u_{2} \in \mathbb{Z}_{p}[[T]]^{\times}$ are invertible power series.

Proof. By proposition 3.1 one easily observes

(i) $f\left(q^{-s}, \gamma, H_{p o s}^{0}\left(X_{\infty}, Z_{p}\right)^{\psi}\right)=\left\{\begin{array}{cc}1 & \text { if } \psi \neq 1 \\ 1-q^{-s} & \text { if } \psi=1\end{array}\right.$ 
(ii) $f\left(q^{-s}, \gamma, H_{p o s}^{2}\left(X_{\infty}, Z_{p}\right)^{\psi}\right)=\left\{\begin{array}{cl}1 & \text { if } \psi \neq \omega^{-1} \\ 1-q^{1-s} & \text { if } \psi=\omega^{-1}\end{array}\right.$

Since $H_{n}$ is $p$-CM we have a decomposition

$$
H_{p o s}^{1}\left(X_{n}, Q_{p} / Z_{p}\right)=H_{e t}^{1}\left(X_{n} \backslash S_{p}, Q_{p} / Z_{p}\right)^{+} \oplus H_{e t}^{1}\left(X_{n}, Q_{p} / Z_{p}\right)^{-}
$$

where the eigenspaces are formed with respect to the $p$-involution $\rho_{p}$. Going to the limit over $n$ and writing $M_{i}$ for $M_{i}(H), i=1,2$, we observe

$$
H_{p o s}^{1}\left(X_{\infty}, Z_{p}\right)^{\psi}= \begin{cases}M_{1}^{\omega \psi}(-1) & \text { if } \psi \text { is } p \text {-odd } \\ M_{2}^{\omega \psi}(-1) & \text { if } \psi \text { is } p \text {-even }\end{cases}
$$

Therefore if the Greenberg conjecture is true then

$$
H_{p o s}^{1}\left(X_{\infty}, Z_{p}\right)^{\psi} \sim 0
$$

if $\psi$ is $p$-even and $\infty$-odd. Further we have by proposition 3.1:

$$
T_{1}\left(H_{p o s}^{1}\left(X_{\infty}, Z_{p}\right)^{\psi}\right) \sim E^{1}\left(T_{1}\left(H_{p o s}^{1}\left(X_{\infty}, Z_{p}\right)(1)\right)^{\psi}=E^{1}\left(T_{1}\left(H_{p o s}^{1}\left(X_{\infty}, Z_{p}\right)^{\omega^{-1} \psi^{-1}}(1)\right)\right.\right.
$$

and therefore

$$
T_{1}\left(H_{p o s}^{1}\left(X_{\infty}, Z_{p}\right)\right)^{\psi} \sim 0
$$

if $\psi$ is $p$-odd and $\infty$-even. For $\psi p$-odd and $\infty$-odd we have:

$$
\begin{aligned}
f\left(q^{-s}, \gamma, M_{1}^{\omega \psi}(-1)\right) & =f\left(q^{1-s}, \gamma, M_{1}^{\omega \psi}\right) \\
& = \begin{cases}\pi^{\delta \omega \psi} v_{\omega \psi}\left(q^{1-s}\right)^{-1} L_{p}(s, \omega \psi) & \text { if } \psi \neq \omega^{-1} \\
\pi^{\delta_{\omega \psi}} v_{\omega \psi}\left(q^{1-s}\right)^{-1} L_{p}(s, \omega \psi)\left(q^{1-s}-1\right) & \text { if } \psi=\omega^{-1} .\end{cases}
\end{aligned}
$$

For $\psi p$-even and $\infty$-even we have

$$
\begin{aligned}
& f\left(q^{-s}, \gamma, E^{1}\left(M_{1}^{\psi^{-1}}\right)\right)=f\left(q^{-s}, \gamma^{-1}, M_{1}^{\psi^{-1}}\right) \\
& =\operatorname{det}\left(-\gamma q^{-s}, M_{1}^{\psi^{-1}}\right) f\left(q^{s}, \gamma, M_{1}^{\psi^{-1}}\right) \\
& = \begin{cases}\pi^{\delta_{\psi^{-1}}} \operatorname{det}\left(-\gamma q^{-s}, M_{1}^{\psi^{-1}}\right) L_{p}\left(1-s, \psi^{-1}\right) v_{\psi^{-1}}\left(q^{s}-1\right)^{-1} & \text { if } \psi \neq 1 \\
\pi^{\delta_{\psi^{-1}}} \operatorname{det}\left(-\gamma q^{-s}, M_{1}^{\psi^{-1}}\right) L_{p}\left(1-s, \psi^{-1}\right) v_{\psi^{-1}}\left(q^{s}-1\right)^{-1}\left(q^{s}-1\right) & \text { if } \psi \neq 1 .\end{cases}
\end{aligned}
$$

Taking into account that $q$ and $\operatorname{det}\left(-\gamma, M_{1}^{\psi^{-1}}\right)$ are principal units we can change the invertible power series $v_{-}(-)^{-1}$ by a suitable factor, finishing the proof of the theorem. 


\section{References}

[DR] Deligne, P. and Ribet, K., Values of abelian L-functions at negative integers over totally real fields, Inv. Math., 59 (1980), 227-286.

[G] Greenberg, R., On the Iwasawa invariants of totally real number fields, Amer. J. Math., 98 (1976), 263-284.

[Ja] Jannsen, U., Iwasawa modules up to isomorphism, Adv. Studies in Pure Math., 17 (1989) 171-207.

[KS] Kraft, J.S. and Schoof, R., Computing Iwasawa modules of real quadratic number fields, Compos. Math., 97 (1995), 135-155.

[S] Schmidt, A., An arithmetic site over the rings of integers of algebraic number fields, Invent. Math., 123 (1996), 576-610.

[T] Taya, H., Computation of $Z_{3}$-invariants of real quadratic fields, Math. Comp., 65 (1996), 779-784.

[Wag 1] Wagstaff, S., Zeros of p-adic L-functions, Math. Comp., 29 (1975), 1138-1143.

[Wag 2] , The irregular primes to 125,000, Math. Comp., 32 (1978), 583-591.

[Wash] Washington, L., Zeroes of p-adic L-functions, Sem. Delange-Pisot, Poitou; Theorie des Nombres Paris 1980/81, Birkhäuser Boston, Basel, Stuttgart; Ed: M.-J. Bertin.

[W] Wiles, A., The Iwasawa-conjecture for totally real fields, Ann. of Math., 131 (1990) 493-540. 